\title{
THE AUTOMATIC LICENSE PLATE RECOGNITION USING FEATURES EXTRACTION AND NEURAL NETWORKS
}

\author{
REEM Q. ABDUlJABBeR ${ }^{1}$ and MOHAMMED A. SHAKIR \\ Dept. of Electrical and computer Engineering, College of Engineering, University of Duhok, \\ Kurdistan Region-Iraq
}

(Accepted for Publication: December 8, 2020)

\begin{abstract}
The automatic license plate recognition (ALPR) system opens the trendy door to the researchers to think, discover techniques and reach to a result for its necessity. The important of the ALPR system is appeared in the transportation for many reasons such as parking, traffic violations and security. The aim of this paper is to suggest a scheme that will extract car number, country and province from the car images. The proposed scheme is based on digital image processing techniques and neural networks. The proposed algorithm is composite of preprocessing and recognition stages. The preprocessing stage includes: locate the car plate region, binarization, enhancement of the image quality, segment the image into the sub-images. The recognition stage will classify and recognize the segmented sub-images as numbers and characters. In this research, the localization is done through normal cross correlation method. The segmentation includes: segment the car plate into three regions, divide the number and separated character into individual and split the connected characters into separated characters are done through suggested algorithms. The recognition is accomplished using the back propagation neural network (BPNN). The recognizer operates on two sets of data. First set of data includes the whole pixels of the sub-images. The second set of data is based on 16 features extracted from the sub-images. A comparison between these two methods is made. The system is experienced on 99 images of Duhok and Erbil provinces, the environment work is done with MATLAB program. The percentage accuracy is: $100 \%, 100 \%$ and $100 \%$ for the localization, distinguish and segmentation respectively. The recognition rate result for the first method is $94.5 \%$ and the second method is $91 \%$.

Keywords: Automatic license plate recognition system; Car plate detection; Normal cross correlation; Morphological operation; Image segmentation; Back propagation neural network.
\end{abstract}

\section{INTRODUCTION}

$\mathbf{T}$ The car is identified for its shape, model and manufacture date. The companies have the ability to produce lots of cars with the same characteristics. This is led to confusion for distinguishing between the cars. So the car plate is utilized as an identification symbol that determines the region and the owner of the vehicle. The license plate is a rectangle piece which is industrialized from metal, carried the engreem1985@gmail.com;

${ }^{1}$ Corresponding author: College of Engineering, University of Duhok, Kurdistan Region, Iraq information (number, county and province) and hung in the front and the back of the vehicle. If the car recorded a traffic violation, made an accident or entered a specific place, the competent employee will treat with the car number and identifies the car's owner.

Since the car numeric are shown in many application and fields. Lots of researchers are promoted to discover an automatic License plate recognition system. it is contributed to simulate human works in realizing the car plate image as mohammed.shakir@uod.ac 
a text and make it easier. The method has many valuable benefits: it saves more time, the system can finish its task in a few seconds while it takes several minutes with a human, it serves economic issue. The cost of this system is equaled to the prices of the perfect camera and a printer. The ALPR system can be employed in the governmental institution parks for: monitoring cars traffic, saving (daily/monthly) income to these institutions and decreasing the cars jam at the institution gates. The system levels are discussed in this research and applied on the one of the license plate (LP) style in the Kurdistan region which is shown in the 'figure (1)'.
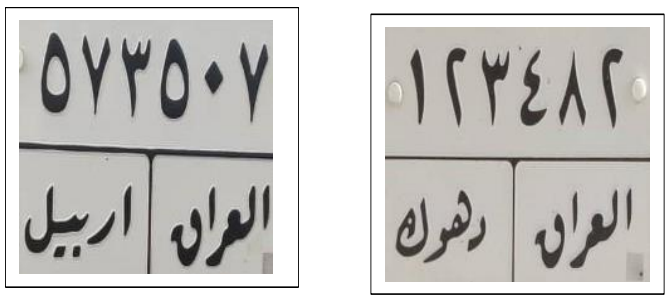

Fig. (1): One of the car plate style in Kurdistan region.

The rest of this section is devoted to review some previous works and to state the contribution of this work to the field of ALPR system.

(Jain, A. S., \& Kundargi, J. M. 2015) proposed an ALPR system, the system algorithm was composed of: preprocessing, LP extract, character segmentation and character recognition. In preprocessing, the noise (impulse, Gaussian and salt and pepper) were removed by median filter and Otsu method binarized the image. The localization was done through: edge detection, morphological operations and connected component analysis techniques. In addition, the angle and area for the car plate were corrected by a specific algorithm. Two stages: preprocessing and character segmentation were utilized in their system to achieve segmentation process based on several methods: quantization, normalization and connected component engreem1985@gmail.com; labeling. Accordingly, the features of the image were extracted then applied on the back propagation neural network to realize the character.

(Wang, J., \& Yan, W. Q. 2016) introduced their system, which has enhanced the image by median filter but the localization was accomplished by horizontal projection method while the vertical projection and statistical techniques were applied to segment the image, finally, the (200) features were taken out from the image by zoning method and fed to the BPNN to recognize the symbol.

(Omran, S. S., \& Jarallah, J. A. 2017, December) suggested the system on the Iraqi cars, it had a new stage which was the 'distinguish between the Iraqi cars styles'. The distinguish algorithm was based on the LP dimensions. In recognition stage the whole segmented image is applied on the BPNN without extracted any symbol feature.

(Sharma, G. 2018), submitted the system on Nepal vehicles. lots of methods were participated for localization and segmentation such as: the edge detection, the morphological operation, the smoothing and the filtering but the recognition were performed by the normal cross correlation.

(Indira, K., Mohan, K. V., \& Nikhilashwary, T. 2019), offered a system for video cars. The system has four stages: car tracking, text extraction, segmentation and recognition. The plate extraction relayed on the 'major pixel transition compared to the pixels surrounding', the vertical projection and templet matching ways were used for the segmentation and recognition, respectively.

In this work, the car plate recognition system is studied and a new scheme for recognition is suggested. the proposed scheme is based on two main stages: preprocessing stage and recognition stage using the back propagation neural network. the new scheme has made modifications to the existing preprocessing techniques. mohammed.shakir@uod.ac 
Two sets of data are applied to the BPNN, at the beginning, the whole image is applied to BPNN and the system performance is tested, secondly, the author suggested an algorithm by which 16 features are extracted from the image. The extracted data are applied to BPNN. A comparison study is made between these two techniques and the operation of the system is verified.

\section{Image processing and neural network:}

As it is known, the execution for ALPR system mostly requires the knowledge in two specifications: digital image processing and artificial intelligent. Thus, the needed techniques for the suggested system are mentioned below:

\subsection{Preprocessing:}

The image processing is aimed to make the processed image more suitable than the original image. The image processing is appeared to reduce the noise, delete the distortion and hide or highlight some attributes in the image (Stastny, Jiri \& Minařík, Martin 2007).

\subsubsection{Normal cross correlation:}

In a general, a correlation is defined as the process of shifting the sub image in the original image and return $\mathrm{C}$ through multiply the overlapped pixels between the template and original images then take the summation for the results. The value which is gotten maximum $\mathrm{C}$ value refers to the site for the best matching. The normal cross correlation is taken due to the correlation is sensitive to the changing of the pixel value (IMAQ, N. 2000). it is useful in template matching and has the equation as shown in the 'equation (1)'.

$$
\mathrm{C}(\mathrm{i}, \mathrm{j})=\frac{\sum_{x=0}^{L=1} \sum_{y=0}^{K=1}(W(x, y)-\bar{W})(f(x+i, y+j)-\bar{f}(i, j))}{\left[\sum_{x=0}^{L=1} \sum_{y=0}^{K=1}(W(x, y)-\bar{W})^{2}\right]^{\frac{1}{2}}\left[\sum_{x=0}^{L=1} \sum_{y=0}^{K=1}(W(x+i, y+j)-f(i, j))^{2}\right]^{\frac{1}{2}}}
$$

Where $\bar{W}$ : the average of the templet pixels value, $\bar{f}$ : the average of the region of the original correspond with templet pixels value and $\mathrm{C}(\mathrm{i}, \mathrm{j})$ : the result for the normal cross correlation and has the range value $(-1,1)$.

\subsubsection{Binarization:}

The image binarization is known a general tool for converting the color of the image to the black and white colors, the reason of using is to separate the object from the background (Jain, R., Kasturi, R., \& Schunck, B. G. 1995). The image is binarized through Otsu method which is segmented the image into two areas: light and dark by putting a threshold to decrease the interfering of the class distributions (Yousefi, J. 2011).

\subsubsection{Image enhancement:}

The noise is considered the first issue for the digital signal because it is caused a distortion in the original signal. The digital photo is two dimensional signal. So it must suffer from it which is appeared as dots, lines and T-shape on engreem1985@gmail.com;

\footnotetext{
${ }^{1}$ Corresponding author: College of Engineering, University of Duhok, Kurdistan Region, Iraq
} 
- The erosion is one of the most important morphological operation. it is gathered between the original image and the structural element to perform the purpose. The erosion effectiveness is appeared by deleting some small isolated characteristics, fracturing a thin portion and decreasing the object size by erode their boundaries (Solomon, C., \& Breckon, T. 2011). Every side of the image is extracted then saved in a variable and erode with diagonal structure element as shown in the 'figure (2)', it is reiterated for several times to clean it out then they are returned to their places in the treated image.

\begin{tabular}{|l|l|l|l|l|l|}
\hline 1 & 0 & 0 & 0 & 0 & 0 \\
\hline 0 & 1 & 0 & 0 & 0 & 0 \\
\hline 0 & 0 & 1 & 0 & 0 & 0 \\
\hline 0 & 0 & 0 & 1 & 0 & 0 \\
\hline 0 & 0 & 0 & 0 & 1 & 0 \\
\hline 0 & 0 & 0 & 0 & 0 & 1 \\
\hline
\end{tabular}

Fig. (2): Shape of the structural element in the proposed system.

\subsection{The features extraction:}

Feature extraction can be expressed by getting a group of characteristics which define the shape of the symbol and taking into consideration: the accuracy and uniqueness. The aim is to conceal the redundant information, it has two methods: structural and statistical, one of the most popular statistical feature extraction methods is the zoning which calculate the amount of pixels statistically in a particular region that are portioned by a grid (Vithlani, P., \& Kumbharana, C. K. 2015), (Omidiora, E. O., Oladele, M. O., Adepoju, T. M., Sobowale, A. A., \& Olatoke, O. A. 2016). In this work, the extract function also based on the zoning technique, the function purpose is to take out the (16) features of the image as a column which are: the summation and the average of the pixels in the first, second, third and fourth quarter, the first and second half vertically and horizontally for the image. The idea for the extraction is figured out in the 'figure (3)'.

engreem1985@gmail.com; mohammed.shakir@uod.ac 


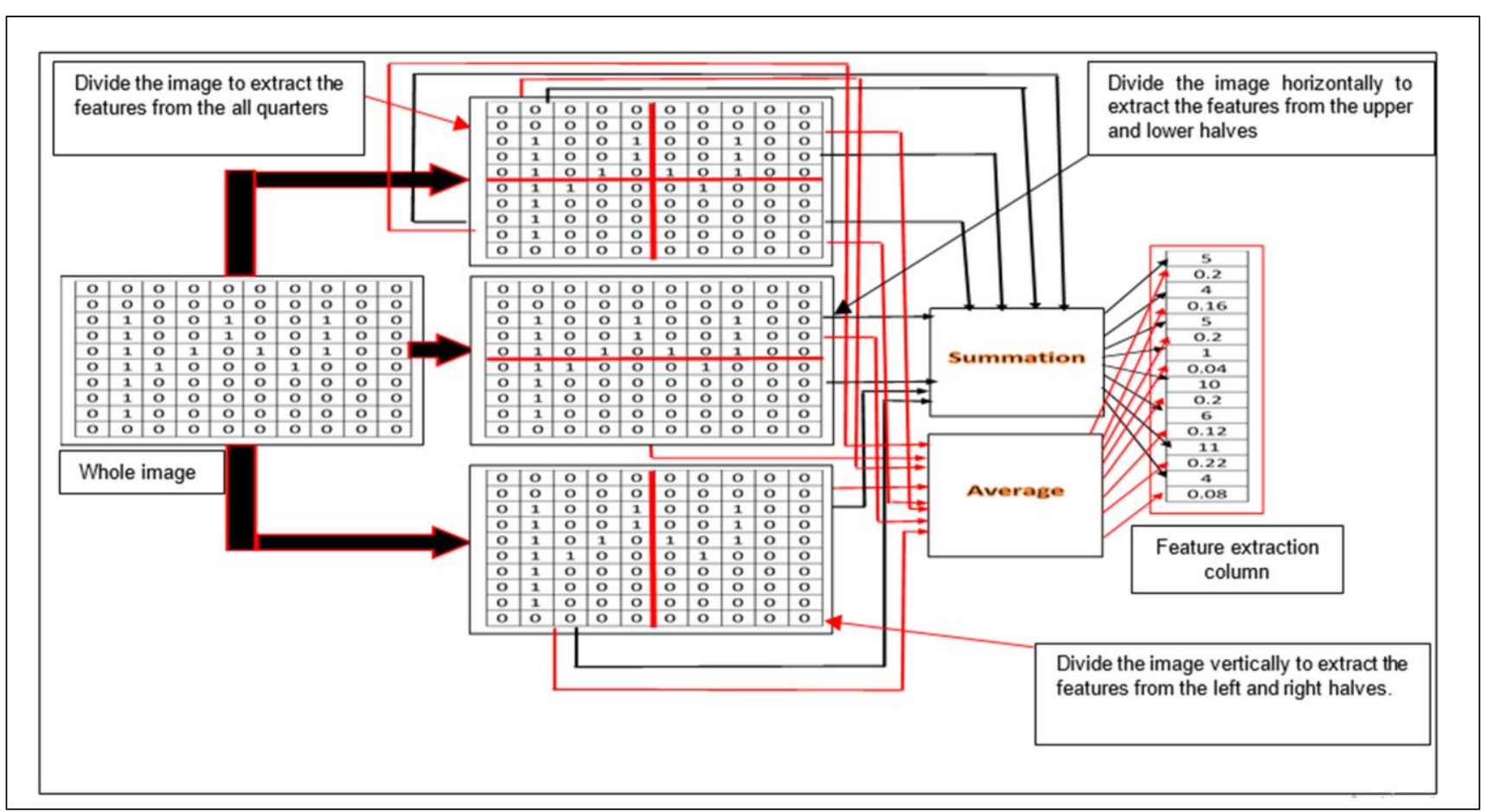

Fig. (3): The feature extraction process in the designed system.

\subsection{Neural Network:}

The neural network technique $(\mathrm{NN})$ has seen a widespread interest, it is utilized in many applications in the medical, engineering, industrial and military sectors. It is discovered for two main reasons: the first reason is knowing how does the human brain work and the second one is to build a machine that can solve a complicated problem which is not possible by the traditional computers. The structure of the
$\mathrm{NN}$ are simulated to the human brain cells in the shape and work, it consists of three layers: input, hidden and output, each layer has the neurons and the connection between layers by the weight as it is shown in the 'figure (4)'.

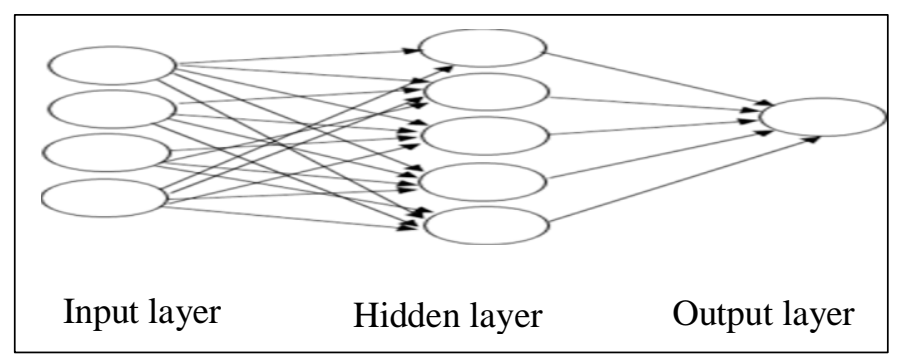

Fig. (4): The shape for the simple neural network.

The NN is provided with the information over the input layer which is fixed.it is sent to the hidden layer which in turn received and

\footnotetext{
${ }^{1}$ Corresponding author: College of Engineering, University of Duhok, Kurdistan Region, Iraq
}

modified then transmitted to the output layer so it is processed in it to produce an output. The number of the neurons in the input and output mohammed.shakir@uod.ac 
layers are depended on the data training while the neurons selection in the hidden layer is deemed as a challenge because if they are increased, the computation will raise whether they are decreased that will prevent the $\mathrm{NN}$ from learning, thus, it should be balance.

The back propagation algorithm is defined as the most popular technique in the neural network.it is based on the mathematical computation to reach the correct output.it consists of four stage: first, feed forward calculation, the back propagation to output layer then to the hidden layer and lastly, weightiness update. The BPNN is stopped from learning when the error become tiny. The features for $\mathrm{NN}$ are: it is a fault tolerance and has the possibility to treat with unclear inputs while the down side are: it takes a lot of time to train data and needs a large amount of data to be efficient (Cilimkovic, M. 2015).

In this paper, Six BPNN are designed for the system, the first case has two networks while the second has four networks. Every network has three layers: input, hidden and output layers, the hidden layer consists of two layers. The numbers in the 'table (1)' refer to the neurons at each layer in every network. The two BPNNs in the first method are used to realize numeric and alpha-numeric images so they have 256 and 450 neurons in their input layers. these values represent the size of the training image (number and character). The size of the number's image is $16 * 16$ while the character's image is $30 * 15$, it is appropriate size and clear to see without any distortion, 10 and 12 neurons in their output layers, respectively. The 10 are chosen due to the range of the recognized numbers are from (0 to 9) while the 12 are selected because it refers to the "دهوك","العراق" characters in the words: and"أربيل". The 20 and 15 neurons in their hidden layers, the number of the layers and neurons depend on the try and error method and to be seen later in section (4).

Table (1): The construction for the designed networks.

\begin{tabular}{|c|c|c|c|c|c|c|c|}
\hline \multirow{3}{*}{\multicolumn{2}{|c|}{ Neurons layers in }} & \multicolumn{2}{|c|}{$\begin{array}{l}\text { The BPNN structure for first method } \\
\text { (Whole image) }\end{array}$} & \multicolumn{4}{|c|}{$\begin{array}{l}\text { The BPNN structure for second method } \\
\text { (Extract image feature) }\end{array}$} \\
\hline & & \multirow[t]{2}{*}{ Recognize number } & \multirow[t]{2}{*}{ Recognize character } & \multirow[t]{2}{*}{ Recognize number } & \multicolumn{3}{|c|}{ Recognize character } \\
\hline & & & & & Iraq & Duhok & Erbil \\
\hline \multicolumn{2}{|c|}{ Input layer } & 256 & 450 & 16 & 16 & 16 & 16 \\
\hline Hidden & First layer & 20 & 20 & 20 & 20 & 20 & 20 \\
\hline Layer & Second layer & 15 & 15 & 15 & 15 & 15 & 15 \\
\hline \multicolumn{2}{|c|}{ Output layer } & 10 & 12 & 10 & 5 & 4 & 5 \\
\hline
\end{tabular}

The difference in BPNN for the second method are: the BPNNs which is used to recognize the character are portioned to three networks because it is faster and give the better result. Every individual word has its own neural network. The 16 neurons represent the extracted image features while in the output layers, the 5 neurons in Iraq network are the unrepeated character in Iraq word, the 4 neurons in the Duhok network are the character in the Duhok word, the 5 neurons in the Erbil network contain 2 neurons from the Iraq network and the rest from the Erbil word. The 'table (2)' displayed the amount of the input images in the networks for the first and second methods.

engreem1985@gmail.com; mohammed.shakir@uod.ac 
Table (2): The number of training image for the BPNNs.

\begin{tabular}{|c|c|c|c|c|c|}
\hline \multicolumn{2}{|c|}{$\begin{array}{l}\text { The BPNN training image for first method } \\
\text { (Whole image) }\end{array}$} & \multicolumn{4}{|c|}{$\begin{array}{l}\text { The BPNN training image for second method } \\
\text { (Extract image feature) }\end{array}$} \\
\hline \multirow[t]{2}{*}{ Recognize number } & \multirow[t]{2}{*}{ Recognize character } & \multirow[t]{2}{*}{ Recognize number } & \multicolumn{3}{|c|}{ Recognize character } \\
\hline & & & Iraq & Duhok & Erbil \\
\hline 1000 & 1500 & 1000 & 600 & 400 & 500 \\
\hline
\end{tabular}

It is necessary to mention that the percentage accuracy (Anand, A. 2017) for the proposed system is computed through 'equation (2)'.

$$
\text { Percentage accuracy }=\frac{(\text { the number } \text { of } \text { correct } \text { images })}{\text { the number of } \text { image }} * 100 \% \text {. }
$$

\section{The proposed system:}

The flowchart of the system is illustrated out in the 'figure (5)', after the image is captured by the smart phone camera from the various places: streets, parks and governmental institutions with a distance one meter from the camera.

engreem1985@gmail.com; mohammed.shakir@uod.ac 


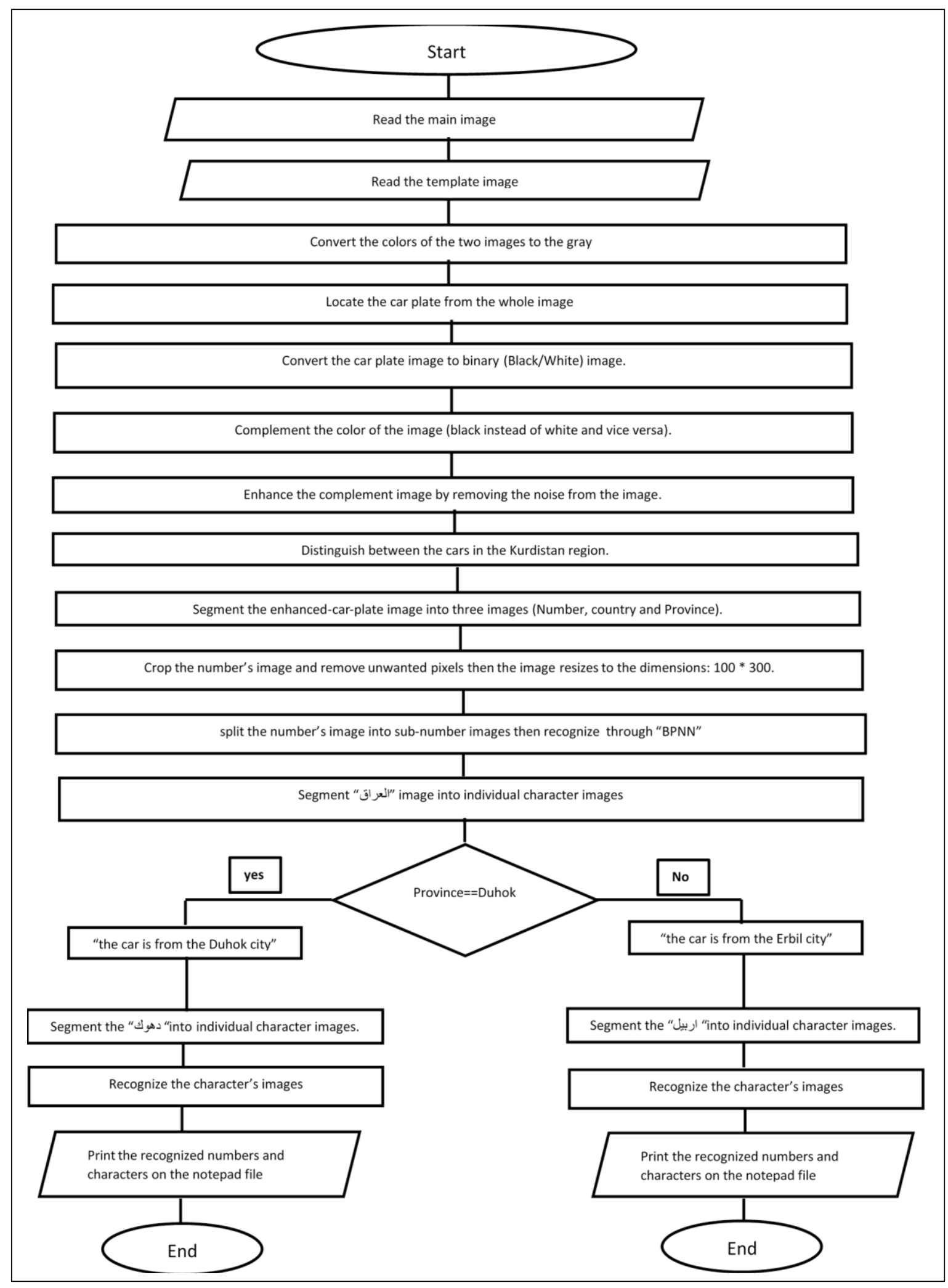

Fig. (5): The structure of the proposed system.

engreem1985@gmail.com; mohammed.shakir@uod.ac $446{ }^{1}$ Corresponding author: College of Engineering, University of Duhok, Kurdistan Region, Iraq 
The car plate region is allocated from the whole image based on the normal cross correlation technique which requires two grayscale images the original image (car) and sub-image (car plate). The best position in area matching will be determined by the normal cross correlation function. The sub image dimensions: width and height are subtracted from the (xpeak, ypeak) of the point to find the beginning area and they are added to the same point to select the ending area then the area is cropped as in the 'figure (6)'.

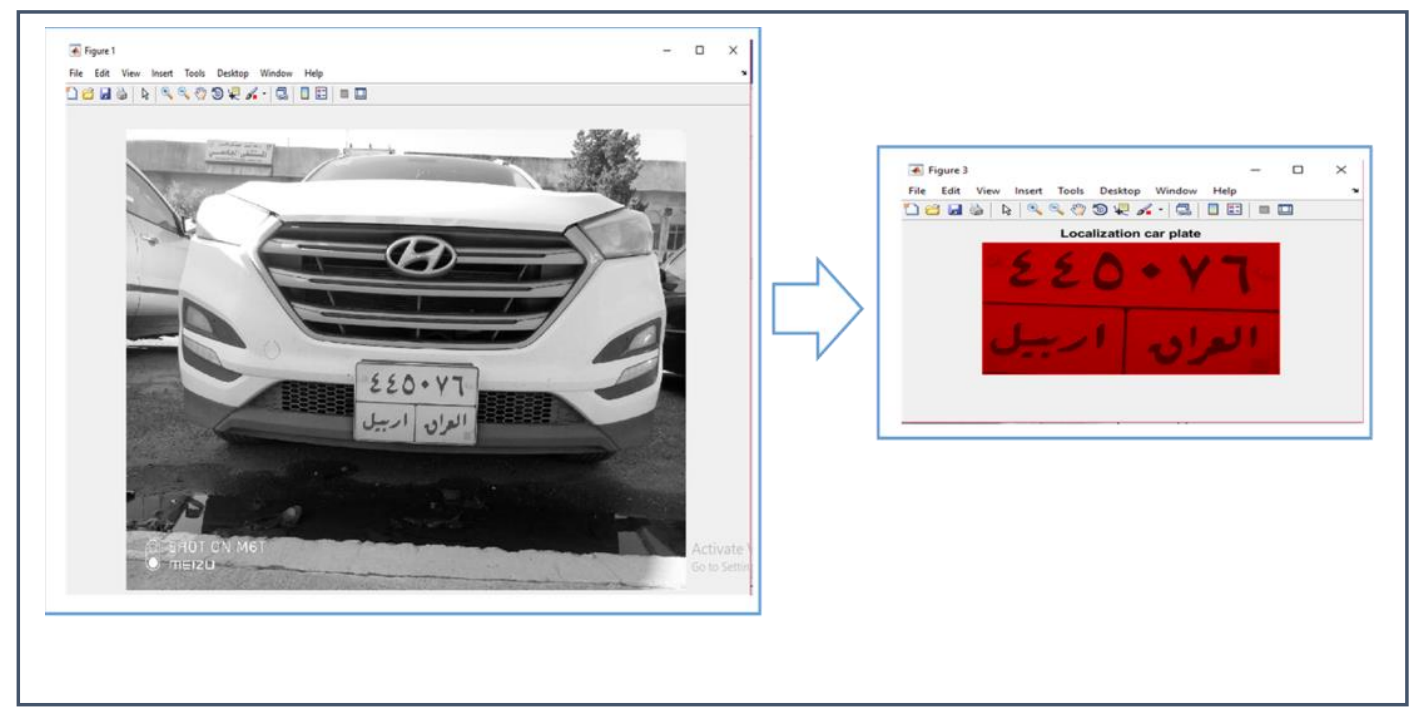

Fig. (6): Localization for the LP from the whole image.

After that, the color image is binarized to the black and white and complemented (the color image is vice versa). Because the noises: dots, lines and T-shape are appeared in the car plate. An enhancement stage is necessary for the suggested system to insure that unwanted pixels are removed and the quality of the image is improved. The enhancement procedure contains these steps: resizing LP image to the dimensions $(256 * 256$, the dots are removed by using connected component analysis and labeling by deleting the area which is less than 70 , the thin and thick lines on the image boundary are canceled through utilizing the morphological operation (erosion) process. The erosion is repeated three times to get good result and finally, the T-shape is available in the LP image to divide it into three regions. The T-shape is considered as a noise by the system, so it was treated by labeling the white area in the image then subtracting the enhanced image from its labeled-area.
Next, it is followed by the flowchart shown in the 'figure (7)' to distinguish between the cars in Kurdistan region which is applied on the cars in the Duhok and Erbil cities. The idea is built on the mathematical fundamental by taking the summation to the four different areas in the left down position from the car plate image, especially, in the province word. The four areas are selected because they contain the maximum height of the Duhok character shape. The areas are: $((35: 40),(50,60),(80,90)$ and $(90,100))$ for the columns with all rows. The operation is implemented on the Duhok's cars and the minimum and maximum summation value are: (80,275), (90,300), (37-87) and (100-230) for the selected areas. After that a new car is experienced if their summation is between the above values, that is mean, the car is from the Duhok city otherwise, it is from the Erbil city. If the car is another type, the flowchart will be expanded in the future work.

engreem1985@gmail.com; mohammed.shakir@uod.ac

\footnotetext{
${ }^{1}$ Corresponding author: College of Engineering, University of Duhok, Kurdistan Region, Iraq
} 


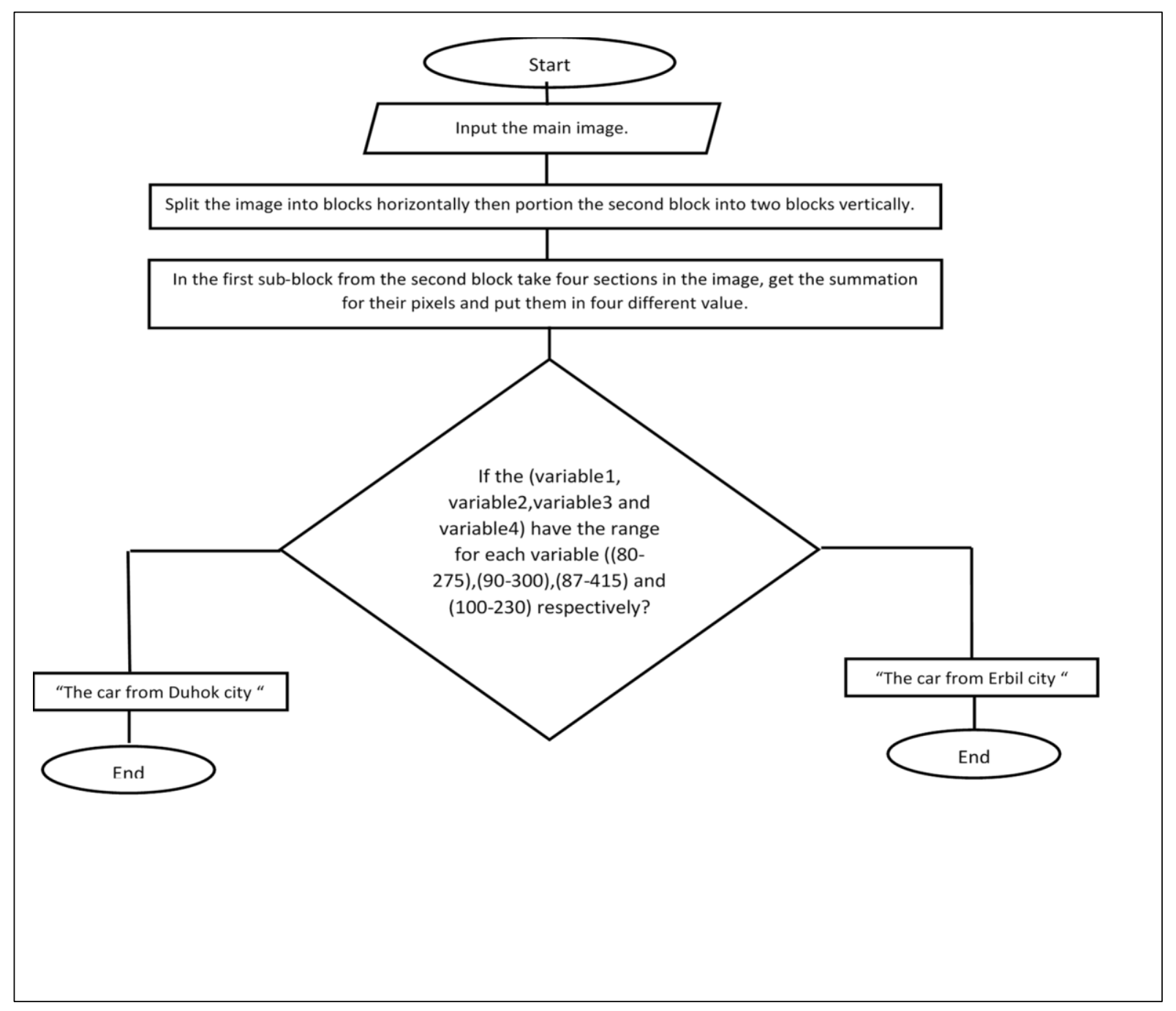

Fi. (7): The distinguish flowchart scheme.

Subsequently, it is the time for segmentation stage which is divided the LP image into the sub-images to prepare them for the next stage. At the beginning, the LP image is partitioned into the three sub images: number, country and province by dividing the car plate into two sectioned into two blocks vertically. Further, the number image is split into individual number sub-images based on the scanning the image column by column, the pixels of each column are compared with the created matrix (one dimension and represented as zeros column), if the pixels did not match, the position's column is saved as a reference for starting the first blocks horizontally then the second block is

number then the scanning is continued from the reference point until the pixels between columns matched, the site of column is stored as a second reference for ending the first number , the two operations are repeated to extract all the numbers from the image.

Lastly, the country and province images are cut up into separated character images, the characters in the words (دهوك","العراق") and "(اربيل") has two styles: separated characters and connected characters. The idea for taking out the separated character is the same for the number while the connected characters' idea is: the extracted section is scanned for several columns, the summation for the pixels' column are engreem1985@gmail.com; mohammed.shakir@uod.ac 
calculated then the position of the maximum summation is keeping as a reference, some pixels are added to it to ensure right cutting, the process is done again till segmenting all characters. The idea for segmentation is demonstrated in the 'figure (8)'.

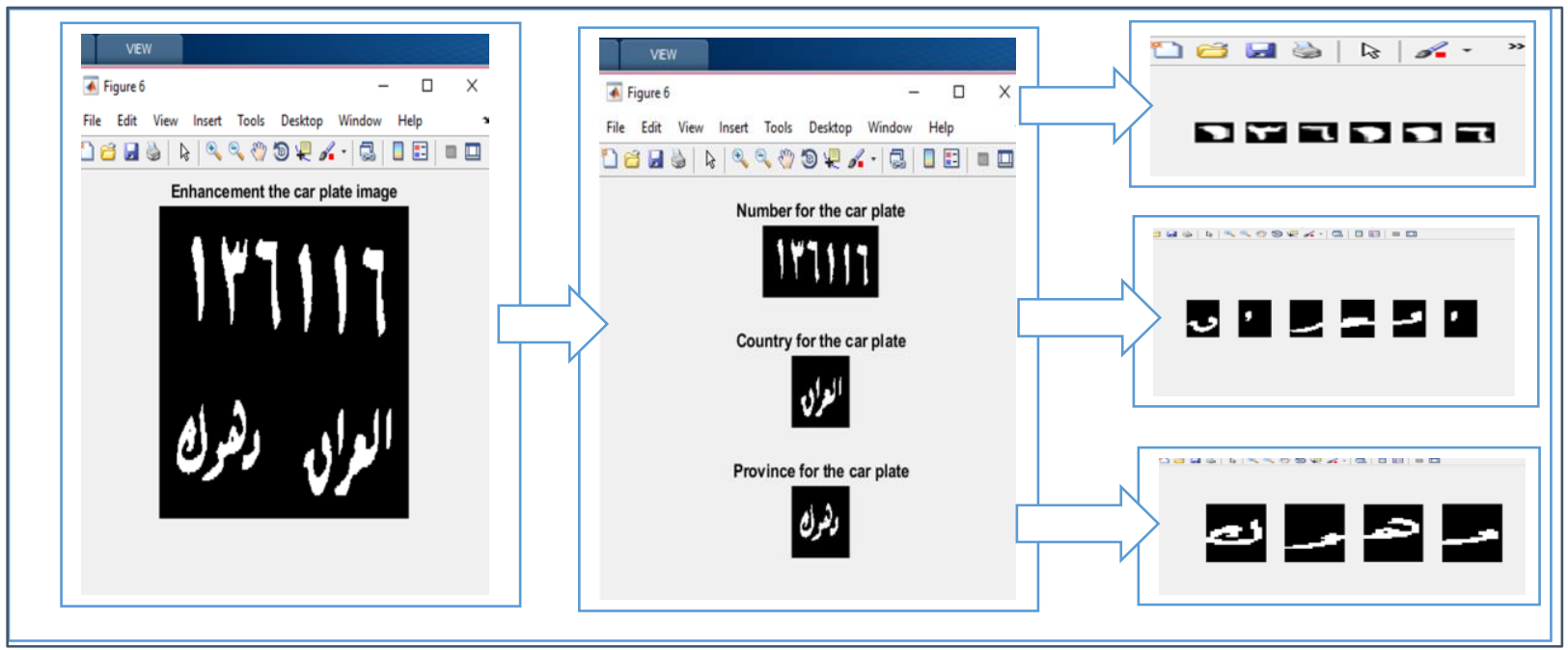

Fig. (8): Demonstration for the segmentation process.

Finally, the segmented images are recognized as a text depends upon the back propagation neural network which is utilized in two ways: firstly, the whole (number/character) image is applied on the network and secondly, the image features are extracted then put on the BPNN. MATLAB program is used to construct the BPNNs through the instruction (patternnet), then the networks are trained the input image with the corresponding output, the percentage for training is $90 \%$ and for testing is $10 \%$, now, every system level is completed and the result of the system is printed in a text file.

\section{RESULTS}

In this research, the various system stages are tested on the 99 car images in the two cities of Kurdistan region in the two cities: Duhok and Erbil. The proposed system is done with MATLAB Program. The individual result for each stage of the proposed system are displayed in the 'table (3)'.

engreem1985@gmail.com; mohammed.shakir@uod.ac

\footnotetext{
${ }^{1}$ Corresponding author: College of Engineering, University of Duhok, Kurdistan Region, Iraq
} 
Table (3): The accuracy of the system level.

\begin{tabular}{|c|c|c|c|c|c|c|}
\hline NO. & \multicolumn{3}{|c|}{ Title of LPR system stage } & System accuracy & \multicolumn{2}{|c|}{$\begin{array}{c}\text { Percentage of } \\
\text { accuracy }\end{array}$} \\
\hline 1 & \multicolumn{3}{|c|}{ Localization for car plate } & $99 / 99$ & \multicolumn{2}{|c|}{$100 \%$} \\
\hline 2 & \multicolumn{3}{|c|}{ Distinguish algorithm } & $99 / 99$ & \multicolumn{2}{|c|}{$100 \%$} \\
\hline 3 & \multicolumn{3}{|c|}{ Segment the car plate into three sub-images. } & $99 / 99$ & \multicolumn{2}{|c|}{$100 \%$} \\
\hline 4 & \multicolumn{3}{|c|}{ Segment the number image to individual images. } & $99 / 99$ & \multicolumn{2}{|c|}{$100 \%$} \\
\hline \multirow[t]{2}{*}{5} & \multirow{2}{*}{$\begin{array}{c}\text { Segment the } \\
\text { ("دهوك"," "العراق") and } \\
\text { ") image to } \\
\text { individual characters. }\end{array}$} & \multicolumn{2}{|c|}{ Separated characters } & $99 / 99$ & \multicolumn{2}{|c|}{$100 \%$} \\
\hline & & \multicolumn{2}{|c|}{ Connected characters } & & & \\
\hline \multirow[t]{4}{*}{6} & \multirow[t]{4}{*}{ Recognition } & \multirow[t]{2}{*}{ Directly } & Numbers & $93 / 99$ & $94 \%$ & $94.5 \%$ \\
\hline & & & Characters & $94 / 99$ & $95 \%$ & \\
\hline & & \multirow{2}{*}{$\begin{array}{c}\text { Feature } \\
\text { extraction }\end{array}$} & Numbers & $91 / 99$ & $92 \%$ & $91 \%$ \\
\hline & & & characters & $89 / 99$ & $90 \%$ & \\
\hline
\end{tabular}

In the previous researches, the normal cross correlation method is utilized in segmentation level while in this research, it is used in the localization process. In this paper, the authors suggested modifications to the existing segmentation and image enhancement techniques. Concerning to the artificial intelligent technique, the back propagation neural network which is particularized as a quick understanding and easy programming, is employed in two modes as it is explained in section (2).
The result for the two methods are compared in the 'table (4)' in term of the following: the amount of neurons in the input layer, the overall system accuracy, the system speed and the best percentage value for training and testing. It is clear that the neurons in first method is more than the second method that is mean the first technique has more computation operation. The accuracy for the first way is better than the second. The structure of the second method is simpler than the first method because it requires less number of neurons and input data set.

Table (4): Comparison between two modes in recognition stage.

\begin{tabular}{clcc}
\hline Number & \multicolumn{1}{c}{ Comparison points } & Directly & Extract image feature \\
\hline 1 & The neurons in the input layer & 256 & 16 \\
\hline 2 & The accuracy of the system & $94.5 \%$ & $91 \%$ \\
\hline 3 & The speed up of the system & Less time & More time \\
\hline 4 & Best percentage value when & Training : $90 \%$ & Training $: 90 \%$ \\
& & Testing : $10 \%$ & Testing $: 10 \%$ \\
\hline
\end{tabular}

'Table (5)' displays the values of the percentage accuracy for the two methods with the variation of (training/testing) ratio. It can be note that increasing the number of the training

engreem1985@gmail.com; ${ }^{1}$ Corresponding author: College of Engineering, University of Duhok, Kurdistan Region, Iraq

samples will improve the percentage accuracy. The best result is obtained when the training and testing are $90 \%$ and $10 \%$ of the input data, respectively.

mohammed.shakir@uod.ac 
Table (5): The system accuracy for the different value of the (training and testing) for two ways.

\begin{tabular}{|c|c|c|c|c|c|}
\hline \multirow[t]{4}{*}{ Number } & \multirow{4}{*}{$\begin{array}{c}\text { Neurons } \\
\text { (one } \\
\text { layer) }\end{array}$} & \multicolumn{4}{|c|}{ Recognition } \\
\hline & & \multicolumn{2}{|c|}{ Directly } & \multicolumn{2}{|c|}{ Extract feature of segment image } \\
\hline & & No. & Char. & No. & Char. \\
\hline & & Percentage for accuracy & Percentage for accuracy & Percentage for accuracy & Percentage for accuracy \\
\hline 1 & 10 & $91 \%$ & $89 \%$ & $91 \%$ & $90 \%$ \\
\hline 2 & 20 & $96 \%$ & $94 \%$ & $93 \%$ & $90 \%$ \\
\hline 3 & 30 & $95 \%$ & $94 \%$ & $86 \%$ & $86 \%$ \\
\hline 4 & 40 & $94 \%$ & $95 \%$ & - & - \\
\hline 5 & 50 & $94 \%$ & $94 \%$ & $\longrightarrow$ & $\longrightarrow$ \\
\hline
\end{tabular}

In the connection with selecting the neurons in the hidden layers, the 'table 6' and 'table 7' demonstrate that best neuron value is (20) if the hidden layer contains one layer and $(20,15)$ if the hidden layer consists of two layers.

Table (6): The best value for neurons if the hidden layer contains one layer.

\begin{tabular}{|c|c|c|c|c|c|c|}
\hline \multirow[t]{4}{*}{ No. } & \multirow[t]{4}{*}{ Training } & \multirow[t]{4}{*}{ Testing } & \multicolumn{4}{|c|}{ Recognition } \\
\hline & & & \multicolumn{2}{|c|}{ Directly } & \multicolumn{2}{|c|}{ Extract feature of segment image } \\
\hline & & & No. & Char. & No. & Char. \\
\hline & & & $\begin{array}{l}\text { Percentage } \\
\text { accuracy }\end{array}$ & $\begin{array}{c}\text { Percentage } \\
\text { accuracy }\end{array}$ & $\begin{array}{l}\text { Percentage } \\
\text { accuracy }\end{array}$ & $\begin{array}{c}\text { Percentage } \\
\text { accuracy }\end{array}$ \\
\hline 1 & 20 & 80 & $82 \%$ & $40 \%$ & $72 \%$ & $88 \%$ \\
\hline 2 & 30 & 70 & $86 \%$ & $66 \%$ & $73 \%$ & $79 \%$ \\
\hline 3 & 40 & 60 & $88 \%$ & $74 \%$ & $80 \%$ & $86 \%$ \\
\hline 4 & 50 & 50 & $85 \%$ & $79 \%$ & $81 \%$ & $88 \%$ \\
\hline 5 & 60 & 40 & $91 \%$ & $87 \%$ & $78 \%$ & $83 \%$ \\
\hline 6 & 70 & 30 & $93 \%$ & $88 \%$ & $83 \%$ & $84 \%$ \\
\hline 7 & 80 & 20 & $94 \%$ & $87 \%$ & $87 \%$ & $89 \%$ \\
\hline 8 & 90 & 10 & $94 \%$ & $95 \%$ & $92 \%$ & $91 \%$ \\
\hline
\end{tabular}

engreem1985@gmail.com; mohammed.shakir@uod.ac 
Table (7): The best value for neurons if the hidden layer contains two layers.

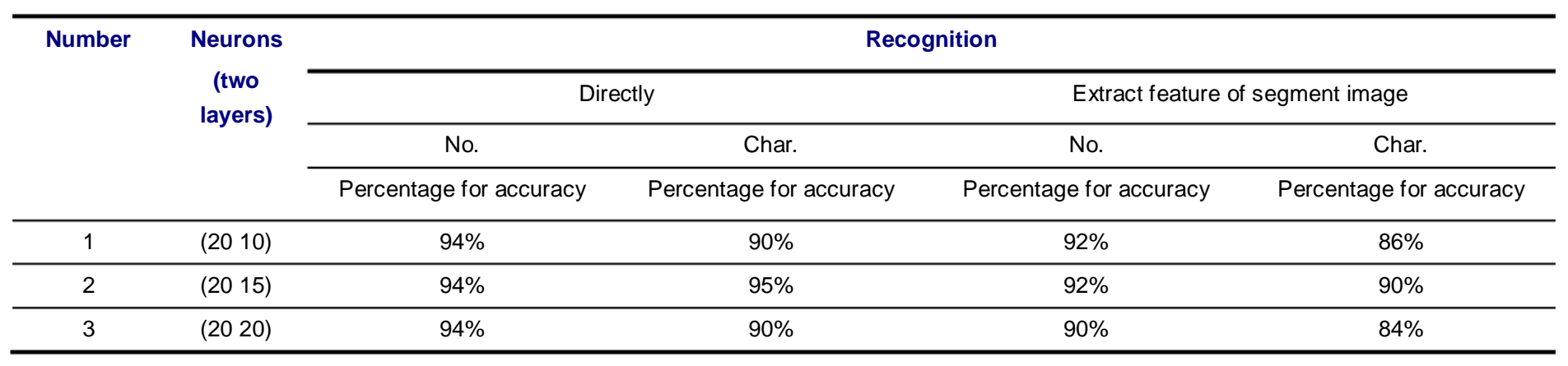

Table (8) is constructed to compare the result of the current research with the previous similar work. The result of the proposed system is slightly better than the result obtained in the (Omran, S. S., \& Jarallah, J. A. 2017, December, b).

Table (8): Result for the proposed system and previous research.

\begin{tabular}{cccc}
\hline \multirow{2}{*}{ Stage } & \multicolumn{2}{c}{ Proposed system } & \multirow{2}{*}{$\begin{array}{c}\text { Pervious research } \\
\text { (Omran, S. S) }\end{array}$} \\
\cline { 2 - 3 } & Directly & Extract feature image & \\
\hline Localization & $100 \%$ & $100 \%$ & $98.3 \%$ \\
\hline Recognition & $94.5 \%$ & $91 \%$ & $93.2 \%$ \\
\hline
\end{tabular}

\section{CONCLUSION}

In this investigation, the aim was to propose an automatic license plate recognition scheme by utilizing sets of digital image processing operations and artificial neural networks: the normal cross correlation, connected component analysis and connected component labeling and morphological operation are employed to extract numbers and characters from the car plate.

Two neural networks are constructed based on the input data. The first one utilizes the whole pixels of sub-images. The second structure uses 16 features extracted from the sub-images. So, two techniques are employed to recognize the numbers and characters.

The results have led to conclude the followings:

- The preprocessing stage is working engreem1985@gmail.com; successfully.

- Increasing the percentage value of training /testing enhances the percentage accuracy.

- The percentage accuracy of the first technique used in recognition is better than the second one.

- The time consumed by the second method is greater than the time taken by first one, due to the required time for extracting the 16 features.

- The data set of the second method is reduced; thus, the complexity of this method is less than from the first one.

\section{REFERENCES}

Jain, A. S., \& Kundargi, J. M. (2015). Automatic number plate recognition using artificial neural network. Int. Res. J. Eng. Technol, 2(4), 633-639.

Wang, J., \& Yan, W. Q. (2016). BP-Neural Network for Plate Number Recognition. International mohammed.shakir@uod.ac 
Journal of Digital Crime and Forensics (IJDCF), 8(3), 34-45.

Omran, S. S., \& Jarallah, J. A. (2017, December). Iraqi License Plate Localization and Recognition System Using Neural Network. In 2017 Second Al-Sadiq International Conference on Multidisciplinary in IT and Communication Science and Applications (AIC-MITCSA) (pp. 73-78). IEEE.

Sharma, G. (2018). Performance analysis of vehicle number plate recognition system using template matching techniques. Journal of Information Technology \& Software Engineering, 8(2), 10-4172.

Indira, K., Mohan, K. V., \& Nikhilashwary, T. (2019). Automatic license plate recognition. In Recent Trends in Signal and Image Processing (pp. 67-77). Springer, Singapore.

Stastny, Jiri \& Minařík, Martin (2007). A Brief Introduction to Image Pre- Processing for Object Recognition.

IMAQ, N. (2000). IMAQ Vision Concepts Manual. National Instruments.

Jain, R., Kasturi, R., \& Schunck, B. G. (1995). Machine vision (Vol. 5, pp. 309-364). New York: McGraw-hill.

Yousefi, J. (2011). Image binarization using Otsu thresholding algorithm. University of Guelph, Ontario, Canada.

Boyat, A. K., \& Joshi, B. K. (2015). A review paper: noise models in digital image processing. arXiv preprint arXiv:1505.03489.

Solomon, C., \& Breckon, T. (2011). Fundamentals of Digital Image Processing: A practical approach with examples in Matlab. John Wiley \& Sons.

Vithlani, P., \& Kumbharana, C. K. (2015). Structural and statistical feature extraction methods for character and digit recognition. International Journal of Computer Applications, 120(24), 0975-8887.

Omidiora, E. O., Oladele, M. O., Adepoju, T. M., Sobowale, A. A., \& Olatoke, O. A. (2016). Comparative analysis of back propagation neural network and self-organizing feature map in estimating age groups using facial features. Current Journal of Applied Science and Technology, 1-7.

Cilimkovic, M. (2015). Neural networks and back propagation algorithm. Institute of Technology Blanchardstown, Blanchardstown Road North Dublin, 15.

Anand, A. (2017). Unit-14 Accuracy Assessment. IGNOU.

engreem1985@gmail.com; mohammed.shakir@uod.ac

${ }^{1}$ Corresponding author: College of Engineering, University of Duhok, Kurdistan Region, Iraq 